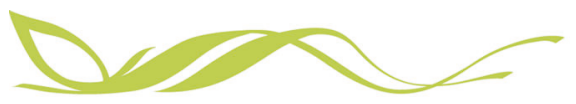

COMMUNICATIONS

ARTICLE

https://doi.org/10.1038/s43247-020-00084-5 OPEN

\title{
Direct measurements of atomic oxygen in the mesosphere and lower thermosphere using terahertz heterodyne spectroscopy
}

Heiko Richter ${ }^{1}$, Christof Buchbender (10 ${ }^{2}$, Rolf Güsten ${ }^{3}$, Ronan Higgins ${ }^{2}$, Bernd Klein ${ }^{3}$, Jürgen Stutzki ${ }^{2}$, Helmut Wiesemeyer ${ }^{3}$ \& Heinz-Wilhelm Hübers (1) $1,4 凶$

Atomic oxygen is a main component of the mesosphere and lower thermosphere of the Earth, where it governs photochemistry and energy balance and is a tracer for dynamical motions. However, its concentration is extremely difficult to measure with remote sensing techniques since atomic oxygen has few optically active transitions. Current indirect methods involve photochemical models and the results are not always in agreement, particularly when obtained with different instruments. Here we present direct measurements-independent of photochemical models - of the ground state ${ }^{3} \mathrm{P}_{1} \rightarrow{ }^{3} \mathrm{P}_{2}$ fine-structure transition of atomic oxygen at 4.7448 THz using the German Receiver for Astronomy at Terahertz Frequencies (GREAT) on board the Stratospheric Observatory for Infrared Astronomy (SOFIA). We find that our measurements of the concentration of atomic oxygen agree well with atmospheric models informed by satellite observations. We suggest that this direct observation method may be more accurate than existing indirect methods that rely on photochemical models.

\footnotetext{
${ }^{1}$ German Aerospace Center (DLR), Institute of Optical Sensor Systems, Rutherfordstraße 2, 12489 Berlin, Germany. ${ }^{2}$ I. Physikalisches Institut der Universität zu Köln, Zülpicher Straße 77, 50937 Köln, Germany. ${ }^{3}$ Max-Planck-Institut für Radioastronomie, Auf dem Hügel 69, 53121 Bonn, Germany. ${ }^{4}$ Department of Physics, Humboldt-Universität zu Berlin, Newtonstraße 15, 12489 Berlin, Germany. ${ }_{\text {email: heinz-wilhelm.huebers@dlr.de }}$
} 
A tomic oxygen extends from about $80 \mathrm{~km}$ to above $300 \mathrm{~km}$ in altitude, but with more than $90 \%$ concentrated between 85 and $125 \mathrm{~km}$ (Fig. 1a). It plays an important role for the energy balance of the mesosphere and lower thermosphere (MLT), because it participates in exothermic chemical reactions and it contributes to radiative cooling ${ }^{1,2}$. The latter occurs mainly via emission from $\mathrm{CO}_{2}$ at $15 \mu \mathrm{m}$ and $\mathrm{NO}$ at $5.3 \mu \mathrm{m}$. Both molecules are excited by collisions with ground state atomic oxygen. In particular, quenching of $\mathrm{CO}_{2}$ vibrational levels by collisions with atomic oxygen is important. Therefore, the knowledge about the distribution of atomic oxygen is crucial for retrieval of the kinetic temperature in the MLT from the $15 \mu \mathrm{m} \mathrm{CO}$ radiance $^{3}$. In addition, direct radiative cooling by atomic oxygen occurs via the fine structure transition from the lowest excited state, ${ }^{3} \mathrm{P}_{1}$, into the ground state, ${ }^{3} \mathrm{P}_{2}$, at $63.2 \mu \mathrm{m}(4.7448 \mathrm{THz})^{4}$. This is the dominant cooling mechanism above $\sim 250 \mathrm{~km}$. In the MLT the lifetime of atomic oxygen in the ${ }^{3} \mathrm{P}_{1}$ state is several hours. Because of this long lifetime it can be transported over large distances before it releases its energy and therefore it might be used as tracer for the dynamical motions, vertical transport, tides, and winds ${ }^{5-7}$. This leads to a strong coupling between the dynamics and the photochemistry in the MLT with the energy being released significantly after and far away from the location, where the UV photon is absorbed. An accurate knowledge of the global distribution of atomic oxygen and its concentration profile as well as diurnal and annual variations are therefore essential for understanding the photochemistry and the energy budget of the MLT.

At the atmospheric conditions of the MLT the fine structure line at $4.7448 \mathrm{THz}$ is thermally Doppler broadened with a line width of $\sim 12 \mathrm{MHz}$ for emission originating at $\sim 100 \mathrm{~km}$ where the density of atomic oxygen is largest. Due to the increasing temperature, emission from the thermosphere is broader $(\sim 25 \mathrm{MHz}$ at altitudes $>300 \mathrm{~km}$ ). Direct observation of this transition requires airborne or space-borne instruments, because absorption by tropospheric water vapor prohibits observation with groundbased instruments. In addition, terahertz $(\mathrm{THz})$ spectrometers are notoriously complex, in particular when it comes to airborne or space-borne applications. The $4.7-\mathrm{THz}$ line has been observed a few times by rocket-borne instruments ${ }^{8}$. More observations with global coverage have been done with the Cryogenic Infrared Spectrometers and Telescopes for the Atmosphere (CRISTA) that flew on the space shuttle in the $1990 \mathrm{~s}^{9,10}$. With this spectrometer atomic oxygen densities have been determined at altitudes from 130 to $175 \mathrm{~km}$, which account for about $20 \%$ of the total atomic oxygen in the MLT. Below $130 \mathrm{~km}$ retrieval was not possible, because of the opacity of the transition and the limb view of CRISTA. Measurements of the fine-structure line have also been made with the Far-InfraRed Spectrometer (FIRS-2), a Fourier transform spectrometer on a high-altitude $(\sim 38 \mathrm{~km})$ balloon ${ }^{11}$. From 1989 to 2003 in total 31 spectra have been obtained and analyzed. But again, the line shape of the transition was not
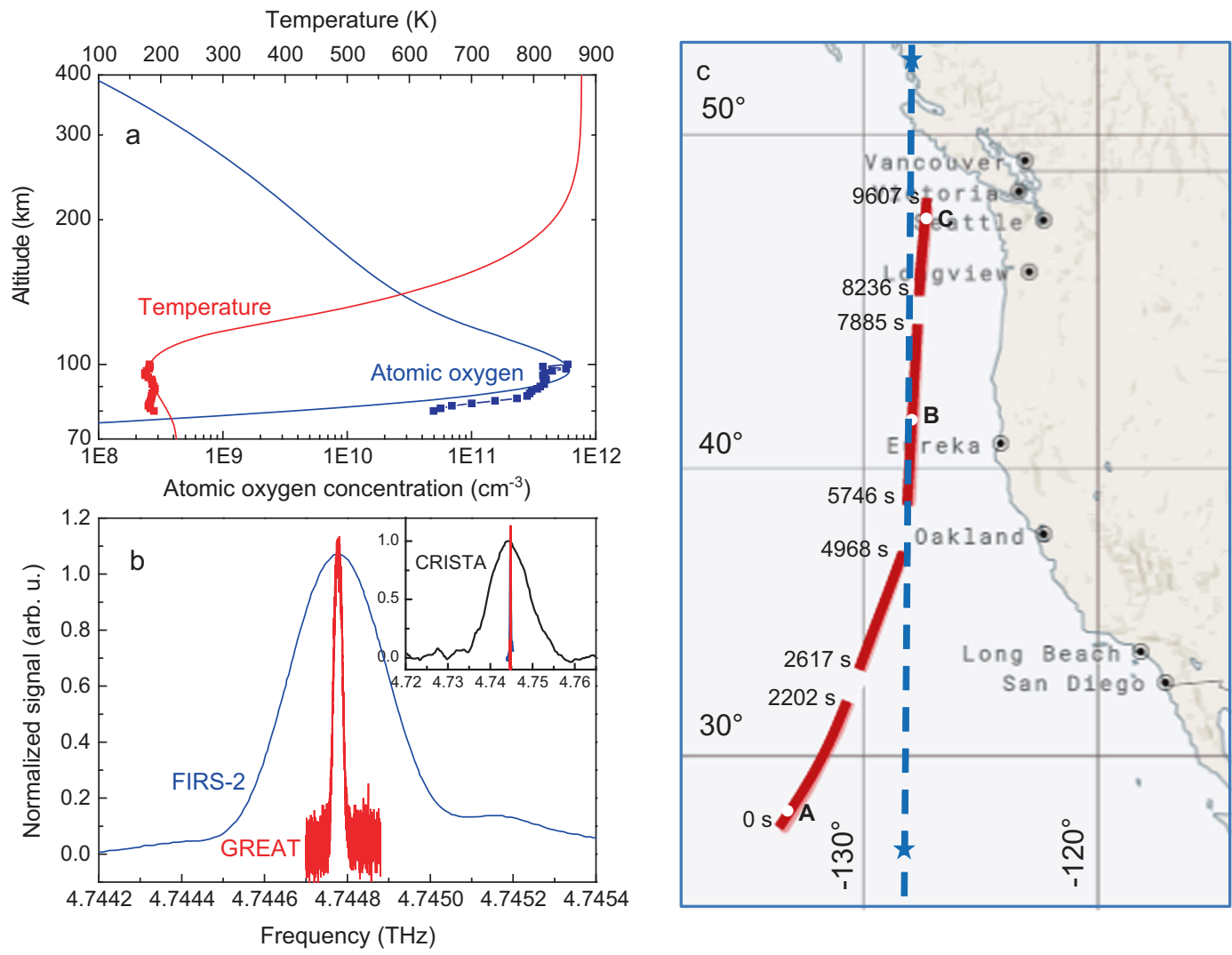

Fig. 1 Atomic oxygen observation with GREAT on SOFIA. a Concentration profile of atomic oxygen and temperature measured by the SABER instrument (blue squares) at altitudes ranging from 80 to $100 \mathrm{~km}$. The straight red and blue lines are calculated profiles with NRLMSISE-00 (at position B in Fig. 1c). b Comparison of the spectra measured with the grating spectrometer of CRISTA (black line in the inset), with the balloon-borne FIRS-2 Fourier transform spectrometer (blue line) and with the GREAT heterodyne spectrometer (red line). Only with GREAT the atomic oxygen line is spectrally resolved. c Trajectory of the SOFIA flight (red line) where the spectra have been acquired between 1:15 and and 4:15 am on 14 January 2015 . The numbers along the trajectory indicate the flight time in seconds. Short interruptions are due to a reorientation of the telescope. The white circles mark the positions where the spectra of Fig. 2 have been measured (A: $50.6^{\circ}, 1: 21 \mathrm{am} ; \mathrm{B}: 38.3^{\circ}, 3: 11 \mathrm{am} ; \mathrm{C}: 29.1^{\circ}, 4: 01 \mathrm{am}$ ). The dashed blue line indicates the flight trajectory of SABER. The red dots and blue squares in $1 \mathrm{a}$ are an average of nine profiles measured with SABER along the flight track between the two blue stars in $1 \mathrm{c}$ on 14 January 2015 between 0:22 and 0:30 am local time (Map: (C) Open Street Map contributors, https://www.openstreetmap.org/copyright). 
resolved. The radiances observed by FIRS-2 deviate by less than $15 \%$ from the computed ones using the Mass Spectrometer Incoherent Scatter (MSIS) model ${ }^{11,12}$.

Atomic oxygen concentrations can be inferred indirectly from measurements of the $\mathrm{O}\left({ }^{1} \mathrm{~S}\right)$ green line, the $\mathrm{O}_{2}$ A-band or the emission from vibrationally excited $\mathrm{OH}$. These methods rely on chemical reaction models and assumptions such as photochemical steady state, quenching rates, radiative lifetimes, and reaction coefficients. The $\mathrm{OH}$ emission has been measured with the SABER (Sounding of the Atmosphere using Broadband Emission Radiometry) infrared spectrometer and with the Scanning Imaging Absorption Spectrometer for Atmospheric Chartography (SCIAMACHY) ${ }^{6,13,14}$. The $\mathrm{O}\left({ }^{1} \mathrm{~S}\right)$ green line was measured by the Wind Imaging Interferometer (WINDII) and SCIAMACHY instruments ${ }^{15-17}$ and the $\mathrm{O}_{2}$ A-band airglow has been measured with SCIAMACHY and the Optical Spectrograph and InfraRed Imaging System (OSIRIS) on the Odin satellite ${ }^{18}$. With all instruments geographical and seasonal variations of the atomic oxygen concentration have been found. Initially, differences up to $60 \%$ are reported for concentrations derived from $\mathrm{OH}$ measurements with SABER and $\mathrm{O}\left({ }^{1} \mathrm{~S}\right)$ measurements with SCIAMACHY ${ }^{16}$. A recent comparison based on an improved photochemical model reveals differences up to $20 \%$ between SABER, SCIAMACHY, WINDII, and OSIRIS measurements ${ }^{19}$. The new atomic oxygen concentrations are about $25 \%$ smaller than those originally derived ${ }^{19,20}$. Likewise, with an improved photochemical model it was found that the atomic oxygen concentrations derived from $\mathrm{OH}, \mathrm{O}\left({ }^{1} \mathrm{~S}\right)$, and $\mathrm{O}_{2}$ A band measurements with SCIAMACHY agree within $15 \%$ with each other, which is better than the agreement with SABER data derived from $\mathrm{OH}$ measurements ${ }^{21}$.

\section{Results and discussion}

Direct observations of atomic oxygen are potentially more accurate. We have analyzed its emission at $4.7 \mathrm{THz}$ measured with the GREAT spectrometer on SOFIA, the Stratospheric Observatory for Infrared Astronomy ${ }^{22,23}$. This is a by-product of the astronomical observations in the same frequency band. In fact, atomic oxygen has been observed in the atmosphere of Mars using GREAT ${ }^{24}$. Due to Doppler shift the line from the astronomical object is often significantly shifted (up to $\sim 3 \mathrm{GHz}$ ) from the atmospheric line. This allows analyzing the spectra of the atomic oxygen line in the Earth atmosphere for those astronomical objects, which are significantly Doppler shifted and not too much broadened, ensuring that the lines do not interfere.

Heterodyne spectroscopy has a number of differences compared to grating spectroscopy and Fourier-transform spectroscopy regarding the measurement of atomic oxygen in the atmosphere. First of all it provides a much higher spectral resolution. In the case of GREAT the spectral resolution of $\sim 6 \mathrm{MHz}$ (full width at half maximum (FWHM), see "Methods" section) is limited by the line width of the local oscillator $(\mathrm{LO})^{25}$. This is significantly smaller than the atomic oxygen line width. In comparison, CRISTA has a spectral resolution of $\sim 4.2 \mathrm{GHz}^{8}$ and FIRS-2 has $120 \mathrm{MHz}$ spectral resolution ${ }^{11}$. The resolving power of GREAT allows spectrally resolving the atomic oxygen line (Fig. 1b). Because of the high spectral resolution the problem of saturation of the atomic oxygen line can be overcome in the vertical sounding observation geometry of GREAT (see "Methods" section). This occurs in the center of the line while the line wings are not saturated and contain information about the atomic oxygen concentration at higher altitudes. The second advantage is the high sensitivity of the GREAT spectrometer. Its singlesideband noise temperature is $2200 \mathrm{~K}$. This corresponds to a noise equivalent power (NEP) of $3 \times 10^{-20} \mathrm{~W}$ within an integration time of $1 \mathrm{~s}$. With this sensitivity GREAT is capable of measuring one spectrum with an integration time of $19 \mathrm{~s}$. For comparison, 31 separate spectra have been measured with FIRS-2 each requiring between 11 and $16 \mathrm{~min}$ of integration time. It should be noted that GREAT is a rather large instrument, which, in its present form, cannot be implemented on a balloon or on a satellite. However, recent improvements in heterodyne technology, in particular high-frequency Schottky diode mixers ${ }^{26}$, compact quantum-cascade lasers as local oscillators ${ }^{27}$, and low-power CMOS (Complementary Metal-Oxide Semiconductor) backend spectrometers ${ }^{28}$ have initiated the development of balloon-borne and satellite instruments ${ }^{29,30}$.

The spectra, which are presented here, have been measured on 14 January 2015 between 1:15 and 4:15 am (flight ID 2015/01/14). The flight trajectory is shown in Fig. 1c. Essentially it is in southnorth direction above the Pacific Ocean west of northern Mexico and California between $27^{\circ}$ and $48^{\circ}$ north latitude and $134^{\circ}$ and $128^{\circ}$ west longitude. The flight direction is from south to north and the telescope is viewing west. Three typical profiles obtained during this flight are shown in Fig. 2. They have been measured at three different elevations, as well as at different locations along the flight track and different altitudes. The measurement uncertainty in intensity is $\pm 15 \%$. It is caused by uncertainties of the absorption by water vapor and calibration uncertainties (see "Methods" section). At the lowest elevation of $29.1^{\circ}$ the profile is the broadest with signs of saturation as the flat top indicates. With increasing elevation it becomes narrower and at the largest elevation of $50.6^{\circ}$ saturation almost disappears, because the path through the atmosphere becomes shorter and less atomic oxygen is probed. It should be noted that most of the signal in the wings of the profile is from altitudes around $100 \mathrm{~km}$. The red solid lines are radiative transfer calculations based on atomic oxygen column densities and temperature profiles from NRLMSISE-00 (US Naval Research Laboratory Mass Spectrometer and Incoherent Scatter Radar Exosphere - 2000) ${ }^{31}$. Each line profile is a convolution with the $6 \mathrm{MHz}$ spectral resolution of the GREAT spectrometer. The elevation of the telescope as well as the absorption by the atmosphere between SOFIA and the MLT are also taken into account (see "Methods" section). The residuals (modeled minus measured data) are up to $15 \%$ and the largest deviations occur in the wings of the profiles, indicating that the atomic oxygen concentration is somewhat different from the model in particular at higher altitudes.

In order to analyze the accuracy of the model we have calculated the emission profiles assuming various deviations from the atomic oxygen concentration and temperature profiles of NRLMSISE-00 (Fig. 3). First, we have varied the concentration profile from 120 to $80 \%$ of the nominal concentration at all altitudes. The residuals show that the best agreement is obtained with the nominal concentrations from NRLMSISE-00. It should be noted that the residuals become more pronounced in the wings of the profile while the peak remains unchanged because of saturation. This underlines the importance of the high spectral resolution. When the temperature, $T$, is changed by $\pm 5 \%$ while the concentration profile is kept nominal the emission becomes much stronger $(T=+5 \%)$ or much weaker $(T=-5 \%)$, because more or less atomic oxygen is excited. An important difference to the residuals for different atomic oxygen concentrations is that in this case the residual at the peak of the line changes significantly. In Fig. $3 c$ calculated profiles are shown which are obtained by shifting the concentration profile by $+5 \mathrm{~km}$ or $-5 \mathrm{~km}$ relative to the nominal profile while keeping the temperature profile unchanged. Again, the deviation from the measured profile is pronounced. When the concentration profile is shifted by $-5 \mathrm{~km}$ a single peak occurs and saturation of the emission is reduced. A double-peak emission profile appears when the concentration 

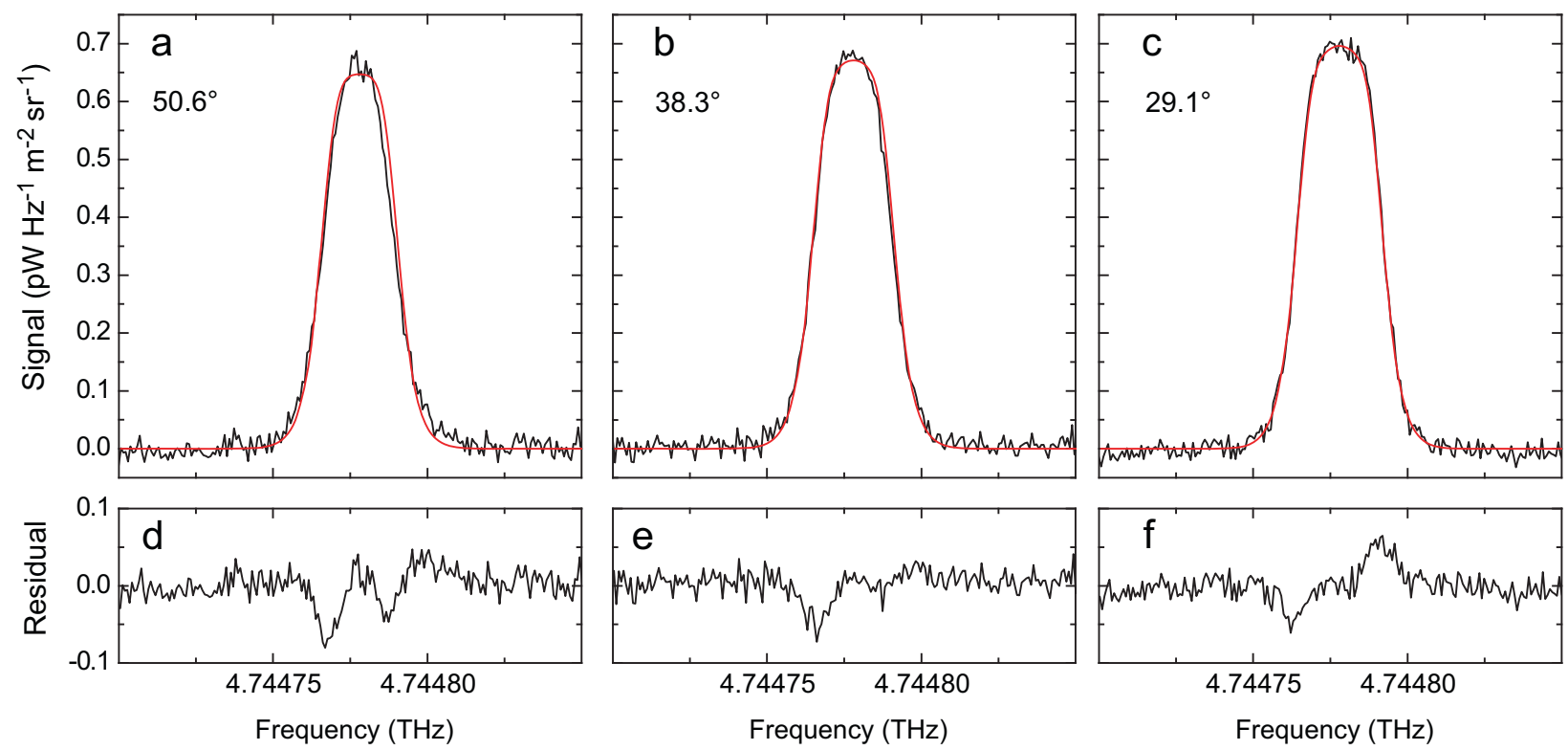

Fig. 2 Emission spectra of atomic oxygen at different elevation of the telescope. a-c Typical spectra of atomic oxygen measured at $50.6^{\circ}, 38.3^{\circ}$, and $29.1^{\circ}$ (black lines, flight altitude: approx. $13 \mathrm{~km}$ ). The red lines are radiative transfer calculations based on NRLMSISE-00. d-f Residuals of the measured and calculated profiles (black lines). With decreasing elevation the saturation of the profile increases, because of the longer optical path through the atmosphere. The positions where the spectra have been measured are marked in Fig. 1c.
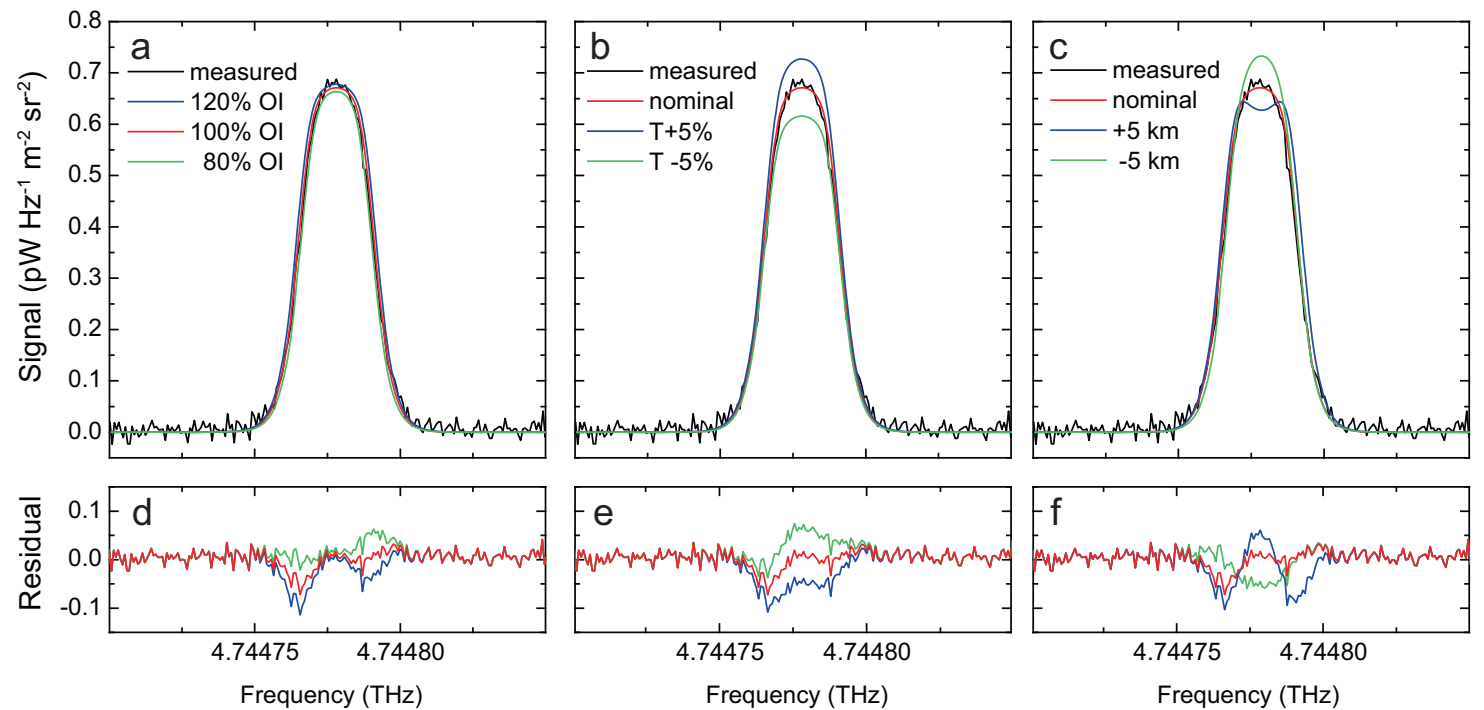

Fig. 3 Dependence of the atomic oxygen emission profile on concentration, temperature and height. a-c Measured spectrum (black line) of atomic oxygen at $38.3^{\circ}$ elevation (same spectrum as in Fig. 2) with several fits (blue, red, and green lines) based on concentration profiles and temperature profiles from NRLMSISE-00. a Atomic oxygen concentration is 120 or $80 \%$ of the nominal value (100\%). $\mathbf{b}$ The temperature profile has been changed by $\pm 5 \%$. In this case large deviations occur at the peak of the line. $\mathbf{c}$ The peak of the nominal concentration profile is shifted by $\pm 5 \mathrm{~km}$. Again, large deviations occur at the peak. d-f The smallest residuals are obtained with the nominal $100 \%$ concentration and the largest deviations are in the wings of the line while the peak is not much affected.

profile is shifted upwards, because the wings of the emission profile are less attenuated. They probe higher atmospheric layers and more atomic oxygen contributes to the signal.

Because NRLMSISE-00 is not a physics-based model, an agreement between this model and the GREAT measurements might be accidental. Therefore we compare the GREAT data with atomic oxygen profiles obtained with the SABER instrument. There is a set of nine SABER profiles, which was measured close to the geolocation and time of the GREAT measurements (see "Methods" section). This data has been measured along the flight track shown in Fig. 1c on 14 January 2015 between 0:22 and 0:30 am local time ${ }^{19}$. (ftp://saber.gats-inc.com/Version2_0/ SABER_atox_Panka_etal_2018_GRL/SABER_o3p_oh_night_ 2015_v1.0.nc). The profiles extend from 80 to $100 \mathrm{~km}$ altitude. Since there are no measured data available for altitudes above $100 \mathrm{~km}$ we have combined SABER and NRLMSISE-00 atomic oxygen and temperature profiles. Below $100 \mathrm{~km}$ these consist of the SABER profile while above $100 \mathrm{~km}$ the NRLMSISE-00 profiles were taken. With these concentration and temperature profiles the expected atomic oxygen emission is calculated and compared with the measured emission (position $\mathrm{B}$ in Fig. 1c). The agreement between the GREAT and the SABER/ 

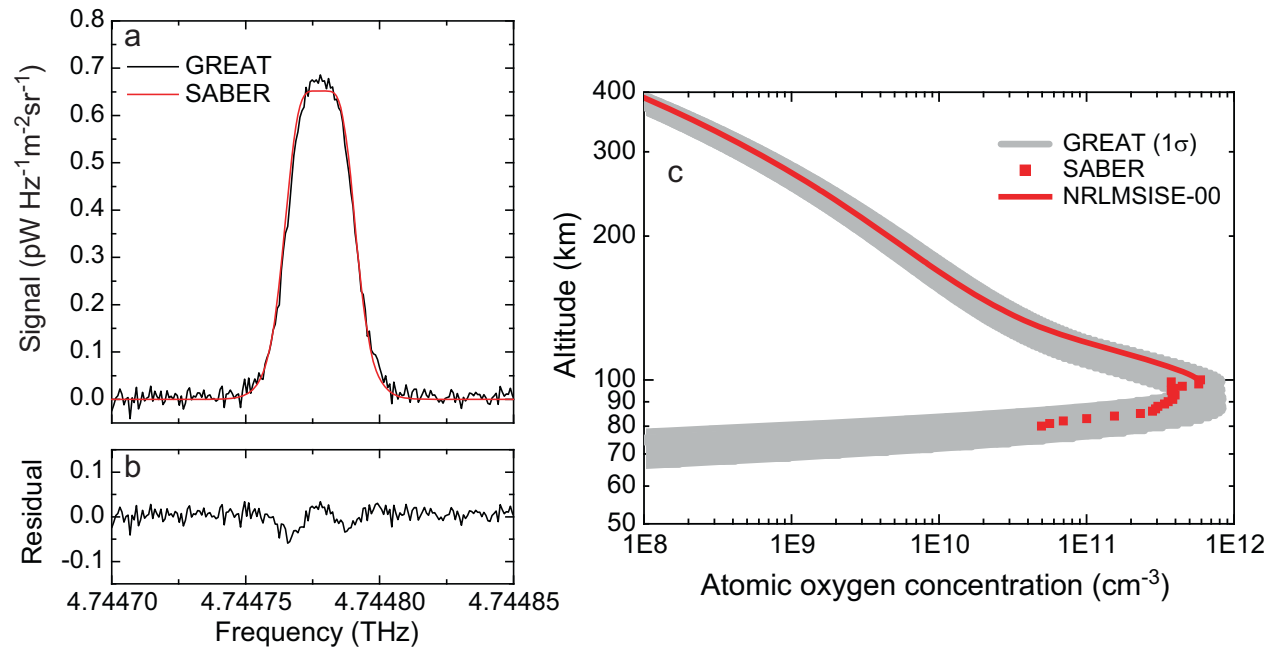

Fig. 4 Atomic oxygen emission and concentration profile. a Emission profile measured with GREAT (black line, position B in Fig. 1c) and calculated emission profile (red line) from combined SABER/NRLMSISE-00 atomic oxygen and temperature profiles. b Residual (black line) of the measured and calculated profiles shown in a. c Combined SABER (red squares) and NRLMSISE-00 (straight red line) profile. The grey area indicates those profiles which are compatible with the measured GREAT spectrum ( $\pm 1 \sigma$ uncertainty).

NRLMSISE-00 profiles is similar as with the pure NRLMSISE00 profiles (Fig. 4a). The differences are within the uncertainty of the GREAT measurement. Also, day-to-day variations of atomic oxygen and temperature as well as uncertainties of the SABER data and NRLMSISE-00 may contribute to the differences. An analysis of the altitude profiles is shown in Fig. 4b. Along with the combined SABER/NRLMSISE-00 profiles it displays a range $( \pm 1 \sigma)$ of atomic oxygen concentration profiles, which are compatible with the GREAT spectra. At the peak this is an uncertainty of approx. $\pm 40 \%$. The range of profiles is derived by varying the atomic oxygen concentration profile, the temperature profile and the relative positon of both starting with the best fit NRLMSISE-00 profile. The variations of these parameters were determined, which provide residuals of less than the measurement uncertainty of $\pm 15 \%$. The combined SABER/NRLMSISE-00 profile falls into this range.

We now turn to the analysis of the flight path in Fig. 1c with 254 spectra. For each spectrum the radiance has been determined by integration of the emission profile and plotted as a function of flight time (Fig. 5). All data are corrected for absorption by atmospheric water vapor, which is determined by fitting the maximum of the saturated emission profiles (see "Methods" section). In contrast to Fig. 2a single value for the precipitable water vapor (pwv) content has been applied to all spectra (see "Methods" section). The calculated radiances take into account the atomic oxygen concentration and temperature profiles from NRLMSISE-00 and the elevation of the telescope. It should be noted that along the flight path the atomic oxygen concentration as well as the temperature predicted by NRLMSISE- 00 change by less than $\pm 5 \%$ at all altitudes between 50 and $400 \mathrm{~km}$. If only the region with the highest concentration of atomic oxygen is considered $(85-125 \mathrm{~km})$ the changes are less than $\pm 2 \%$. The measured radiances agree with the calculated ones within the measurement uncertainty of $\pm 15 \%$. The radiance increases by about $20 \%$, because the elevation of the telescope decreases leading to a longer path through the atmosphere and as a consequence to broader emission profiles and increased radiances (cf. Fig. 2). The shapes of the measured emission profiles along the flight path of SOFIA agree well with the predictions by NRLMSISE-00 and the corresponding atomic oxygen concentration profiles are well within the uncertainty of the GREAT measurement shown in Fig. 4b. It is worth noting that out of the

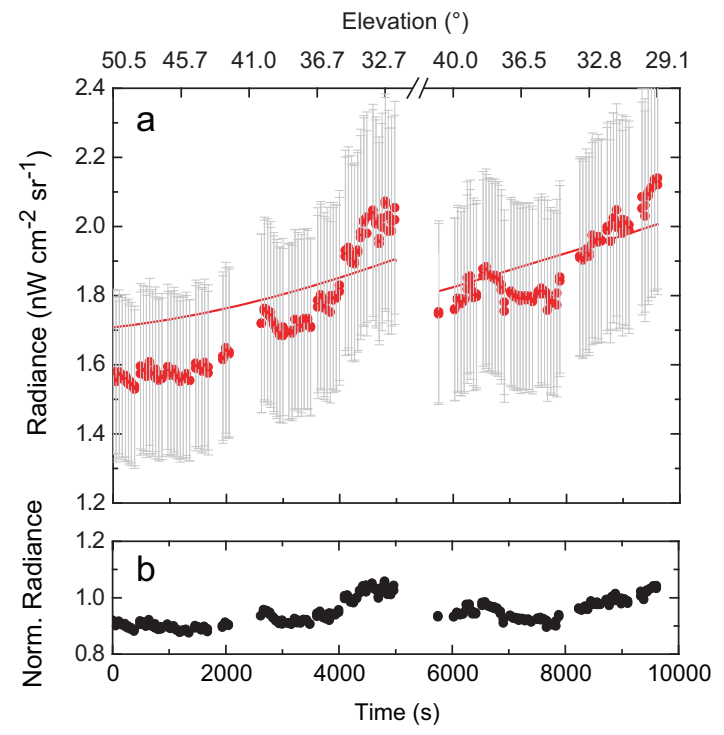

Fig. 5 Radiances measured along the flight path of SOFIA. a Measured (red dots) and calculated (straight red line) radiances along the flight path shown in Fig. 1c (bottom scale: time of flight, top scale: elevation of the telescope). The measured data are corrected for absorption by atmospheric water vapor using the same pwv value for all data. The straight line has been calculated with the NRLMSISE-00 model taking into account the elevation of the telescope. Data obtained from 254 spectra are plotted. The radiance increases until $t=5000 \mathrm{~s}$ and from $t=6000 \mathrm{~s}$ onwards, because the elevation of the telescope decreased from $\sim 51^{\circ}$ to $\sim 32^{\circ}$ (until $5000 \mathrm{~s}$ ) and from $\sim 42^{\circ}$ to $\sim 29^{\circ}$ (from 6000-10,000 s). The interruption between 5000 and 6000 s corresponds to a reorientation of the telescope. b Normalized radiance (black dots, measured radiance divided by calculated radiance) during flight. The measurement uncertainty indicated by the grey bars is $\pm 15 \%$.

31 FIRS-2 radiances 26 are in the range of our measurement between 1.5 and $2.2 \mathrm{nW} \mathrm{cm} \mathrm{cr}^{-2} \mathrm{sr}^{-111}$.

The first spectrally resolved, direct measurement of atomic oxygen in the MLT through its fine-structure transition at 4.7 THz is presented. This is a very promising alternative to established indirect methods which rely on of photochemical 
models. It is an important step towards a conclusive understanding of the photochemistry, energy balance, and dynamics of the Earth atmosphere. This will be substantiated once the large amount of atomic oxygen data, which has been obtained with SOFIA, is completely analyzed. With the recently installed sevenpixel upGREAT heterodyne spectrometer even more data will be available. In particular, the fine-structure transition at $2.06 \mathrm{THz}$ can be measured simultaneously, enabling measurements of wind speed and temperature profile. Beyond that, with the current progress in $\mathrm{THz}$ technology balloon-borne and space-borne heterodyne spectrometers become feasible. Combining such a $\mathrm{THz}$ spectrometer with optical instruments similar to SABER or SCIAMACHY will be even more advantageous for the determination of atomic oxygen in the MLT.

\section{Methods}

The emission of the atomic oxygen fine structure line at $4.7448 \mathrm{THz}$ is observed with GREAT, the German Receiver for Astronomy at Terahertz Frequencies. GREAT is regularly operated on board of the Stratospheric Observatory for Infrared Astronomy (SOFIA) ${ }^{22}$, which is a Boeing $747 \mathrm{SP}$ with a $2.5-\mathrm{m}$ diameter telescope in the rear. The elevation of the telescope can be varied from about $20^{\circ}$ to $60^{\circ}$ and its azimuth is determined by the aircraft heading. At $100 \mathrm{~km}$ altitude the area of the sampled volume is about $5 \mathrm{~m}$ wide and $5 \mathrm{~km}$ long and the distance between two sampled volumes is approximately $15.5 \mathrm{~km}$. This is determined by the spatial resolution of the $2.5-\mathrm{m}$ diameter telescope of SOFIA, which has a diffraction limited beam width of $6 \operatorname{arcsec}$ (FWHM), by the speed of the aircraft, and the integration time for one spectrum, which is $19 \mathrm{~s}$.

GREAT is a heterodyne spectrometer with two frequency channels, one of them at $4.7 \mathrm{THz}$. The observing altitude of SOFIA is $12-14 \mathrm{~km}$, meaning that the emission from atomic oxygen in the MLT will be present in nearly all of the astronomical atomic oxygen observations. Due to the velocity of astronomical objects relative to the Earth the atomic oxygen emission from these objects is usually Doppler shifted relative to the line in the Earth's atmosphere. Thus the astronomical data nearly always contain the atomic oxygen line originating from the MLT. The GREAT heterodyne spectrometer relies on a hot-electron bolometer as mixer and a quantum-cascade laser (QCL) as local oscillator (LO) ${ }^{25,32}$. It has single-sideband noise temperature of $2200 \mathrm{~K}$ enabling a measurement time of $19 \mathrm{~s}$ per spectrum and an intermediate bandwidth ranging from 0.2 to $2.5 \mathrm{GHz}$. The backend spectrometer is a digital fast Fourier transform spectrometer ${ }^{33}$. Its channel spacing is $76.3 \mathrm{kHz}$, more than sufficient for the present observations, where we, in fact, used channel binning down to effectively $0.763 \mathrm{MHz}$ channels. The spectral resolution is ultimately limited by the emission linewidth of the QCL LO, which is Gaussian-shaped with $6 \mathrm{MHz}$ FWHM. The spectral resolution of the GREAT instrument has been determined prior to the flight in the laboratory be measuring reference spectra of $\mathrm{CH}_{3} \mathrm{OH}$ at a pressure of $1 \mathrm{hPa}$. From the analysis of these spectra a spectral resolution of $6 \mathrm{MHz}$ (FWHM) was determined. This linewidth is taken into account when comparing the measured radiances with our radiative transfer model.

For radiometric calibration the GREAT spectrometer is equipped with two blackbody calibration sources. One has a temperature $T_{\text {hot }}$ of $294 \mathrm{~K}$ while the other one is cooled providing a temperature $T_{\text {cold }}=149 \mathrm{~K}$. A measurement cycle for the atmospheric atomic oxygen line is as follows: First, the signals $C_{\text {hot }}$ and $C_{\text {cold }}$ from the hot calibration source and the cold calibration source, respectively, are measured. Then the signal of the sky, $C_{\text {sky }}$, with no astronomical source in the fieldof-view of the telescope is measured. The calibrated signal, $S_{\text {cal }}$, is determined according to

$$
S_{\text {cal }}\left(T_{\text {sky }}, v\right)=\frac{C_{\text {sky }}-C_{\text {hot }}}{C_{\text {hot }}-C_{\text {cold }}}\left(B\left(T_{\text {hot }}, v\right)-B\left(T_{\text {cold }}, v\right)\right)+B\left(T_{\text {hot }}, v\right) .
$$

Here, $B\left(T_{\text {hot }}, v\right)$ and $B\left(T_{\text {cold }}, v\right)$ are the radiances of the hot and cold calibration source.

For analysis of the spectra we have implemented a radiative transfer code. In this code the atmospheric radiance is modelled by evaluating the following radiative transfer equation

$$
R_{\mathrm{int}}=\iint_{\nu, z} B_{\nu}(T(z)) \frac{\partial \tau_{\nu}}{\partial z} \mathrm{~d} z \mathrm{~d} \nu,
$$

with $R_{\text {int }}$ being the spectrally integrated radiance $\left(\mathrm{nW} \mathrm{cm}{ }^{-2} \mathrm{sr}^{-1}\right) . T$ is the temperature at altitude $z$ and $v$ is the frequency. $B_{v}(T(z))$ is the source function at altitude $z$. As has been shown in previous studies, local thermodynamic equilibrium (LTE) determines the population of atomic oxygen up to at least $350 \mathrm{~km}^{34}$. This is in agreement with FIRS-2 measurements, which show that more than $80 \%$ of the atomic oxygen radiance originates from below $\sim 140 \mathrm{~km}$ and its distribution is well modelled assuming LTE ${ }^{11}$. Therefore $B_{v}(T)$ is the Planck blackbody function. The monochromatic transmission $\tau_{v}$ is given by

$$
\tau_{\nu}=\exp \left(-\sigma_{\nu} u\right)
$$

Here $\sigma_{v}$ is the absorption cross section and $u$ is the optical mass along the line of sight. The absorption cross section is the product of the line strength $S$ and the line profile of the atomic oxygen transition. Due to the low pressure this is a Doppler profile. At $4.7448 \mathrm{THz}$ the FWHM of a Doppler profile varies from $12 \mathrm{MHz}$ at $200 \mathrm{~K}$ (corresponding altitude approx. $100 \mathrm{~km}$ ) to approx. $25 \mathrm{MHz}$ at $850 \mathrm{~K}$ (corresponding altitudes $>300 \mathrm{~km}$ ). The line strength $S$ depends on the temperature $T$. At $T_{\text {ref }}=296 \mathrm{~K}$ it is $S=1.131 \times 10^{-21} \mathrm{~cm}^{-1}$ atom ${ }^{-1} \mathrm{~cm}^{2}$ ) and can be calculated for other temperatures according to

$$
S(T)=S\left(T_{\text {ref }}\right) \frac{Q\left(T_{\text {ref }}\right)}{Q(T)} \frac{\exp \left(\frac{-E_{\mathrm{L}}}{k T}\right)}{\exp \left(\frac{-E_{\mathrm{L}}}{k T_{\text {ref }}}\right)} \frac{1-\exp \left(\frac{-h}{k T}\right)}{1-\exp \left(\frac{h}{k T_{\text {ref }}}\right)}
$$

Here $v$ is the frequency, $Q$ is the electronic partition function and $E_{\mathrm{L}}$ is the energy of the lower state of the transition. $E_{\mathrm{L}}$ is zero because the ${ }^{3} \mathrm{P}_{2}$ is the ground state.

To calculate the radiance the atmosphere is divided into horizontally homo-

geneous spherical layers with a thickness $d=1 \mathrm{~km}$ starting at an altitude of $400 \mathrm{~km}$ and ending at $50 \mathrm{~km}$. Absorption and scattering have been neglected. In each layer the transmission $\tau_{v}$ is calculated assuming an average temperature within the layer, which is taken from the NRLMSISE-00 temperature profile ${ }^{31}$. In each layer a portion of the incoming radiation is absorbed which is taken into account by an attenuation factor $\exp \left(-\alpha_{v} l\right)\left(\alpha_{v}\right.$ : absorption coefficient at frequency $v, l$ : absorption length). The radiation which is thermally emitted by the layer is added. The absorption path length takes into account the elevation $\varphi$ of the telescope.

Our radiative transfer code evaluates the radiance across the atomic oxygen line with a step size of $0.763 \mathrm{MHz}$. This ensures that the line profile is properly sampled with at least 20 spectral bins. The monochromatic radiances are integrated in order to obtain the total, integrated radiance (cf. Eq. 2). The integration is done from

$-35 \mathrm{MHz}$ to $+35 \mathrm{MHz}$ around the line center frequency. We have validated our radiative transfer code by calculating the radiances reported in the ref. ${ }^{11}$. As input we used the concentration profiles and temperature profiles provided by NRLMSISE-00. The integrated radiances calculated with our code agree within $1 \%$
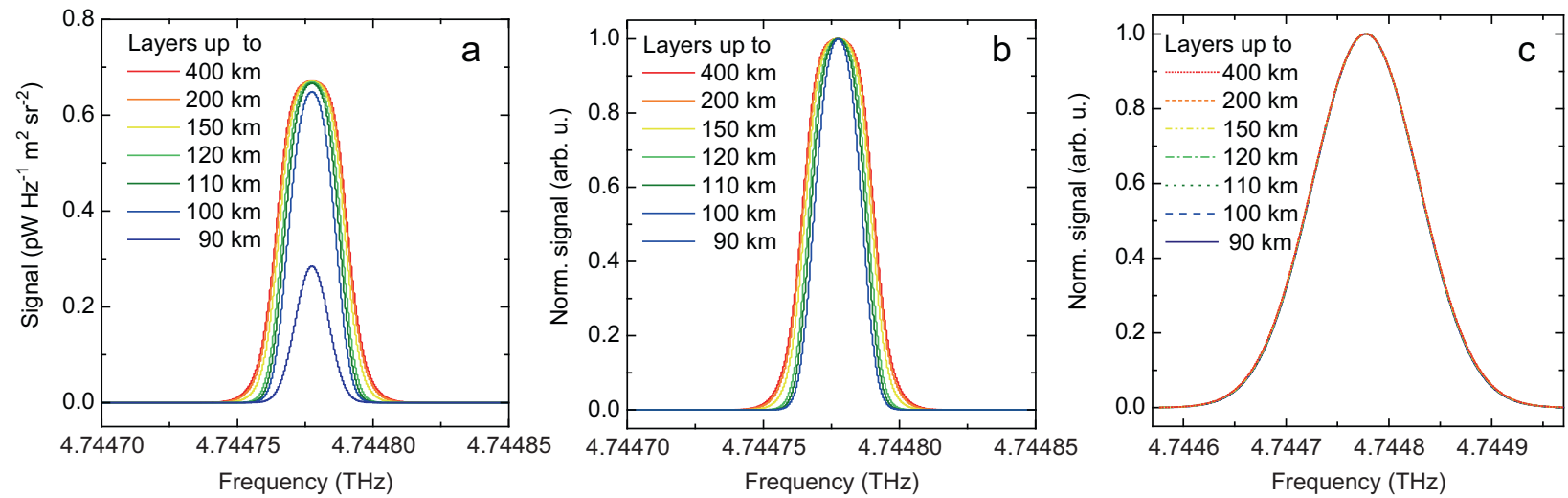

Fig. 6 Calculated emission profiles of atomic oxygen. The calculation is based on NRLMSISE-00 atomic oxygen and temperature profiles taking into account only atmospheric layers up to the value given in the legend. a Convolution with a 6-MHz Gaussian profile corresponding to the resolution of the GREAT spectrometer. b Same as a but all spectra are normalized to 1. c Same as b but the convolution is done with a $120 \mathrm{MHz}$ wide Gaussian profile. In this case the emission profile is not spectrally resolved. 
with the radiances calculated by the radiative transfer code of ref. ${ }^{11}$, which in turn agrees within 1\% with the Full Transfer by Ordinary Line-by-Line Methods (FUTBOLIN) radiative transfer code ${ }^{35}$ and the Monochromatic Radiative Transfer Algorithm (MRTA) ${ }^{36}$.

Figure 6 displays a comparison of the lines shapes simulated with the radiative transfer code assuming a spectrometer with a spectral resolution of $6 \mathrm{MHz}$ (such as GREAT), and an instrument which is not capable to spectrally resolve the atomic oxygen emission (spectral resolution of $120 \mathrm{MHz}$ such as FIRS-2). The spectra are calculated based on atmospheric layers up to a maximum altitude. The highresolution spectra start to saturate in the center of the line when atmospheric layers above an altitude of approximately $110 \mathrm{~km}$ are taken into account (Fig. 6a). However, the wings of the emission line become wider when atmospheric layers from higher altitudes are included in the calculation. This demonstrates that atomic oxygen lines observed with a high-resolution instrument such as GREAT contain information from all altitudes. Figure $6 \mathrm{~b}$ displays the same spectra but normalized in order to better visualize the wings of the profiles, which becomes wider the more atmospheric layers are taken into account. In contrast the line shape observed with
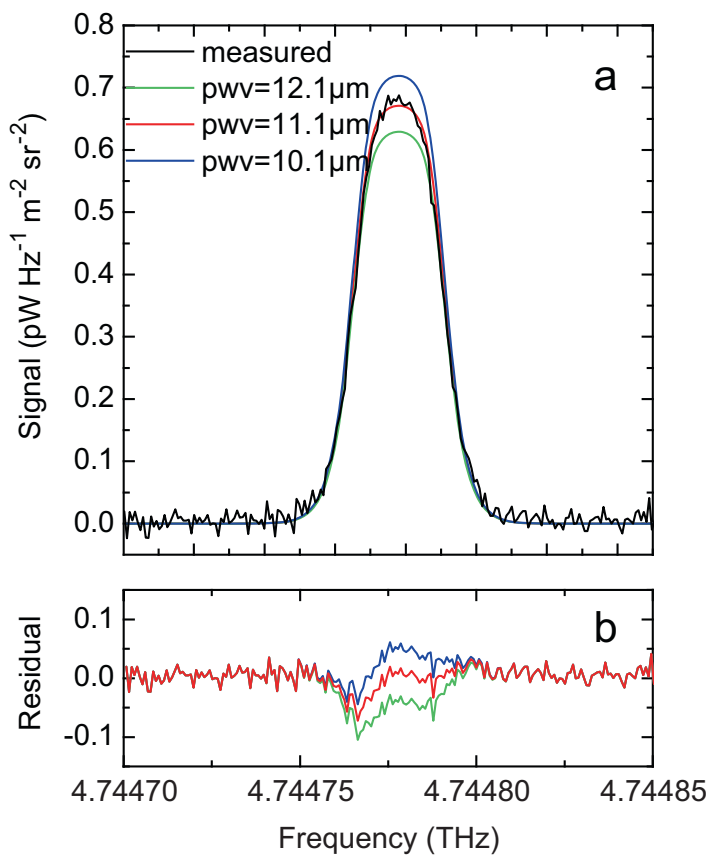

Fig. 7 Influence of the pwv on the emission profile of atomic oxygen. a Measured spectrum of atomic oxygen at $38.3^{\circ}$ elevation (black line, same as in Figs. 2, 3) with several fits based on NRLMSISE-00 with the nominal atomic oxygen and temperature profiles used in Fig. $2 \mathrm{~b}$. The pwv has been changed by $\pm 1 \mu \mathrm{m}$ (green line: $12.1 \mu \mathrm{m}$ pwv, red line: $11.1 \mu \mathrm{m}$ pwv, blue line: $10.1 \mu \mathrm{m}$ pwv). b Residual of measured and calculated emission profile (color code is the same as in $\mathbf{a}$ ). Note that the residual changes mostly at the peak of the line. a low-resolution instrument is entirely determined by the resolving power of the instrument and independent of the atmospheric layers, which are included in the calculation (Fig. 6c).

In order to compare the calculated radiance with the measured radiance the absorption by water vapor in the tropopause and lower stratosphere above SOFIA has to be taken into account ${ }^{37}$. It depends on the precipitable water vapor (pwv) in the atmosphere above SOFIA. From fitting the line profiles in Fig. 2 we obtain pwv values of $14.2 \mu \mathrm{m}$ (elevation: 50.6 $\left.6^{\circ}\right) 11.1 \mu \mathrm{m}\left(38.3^{\circ}\right)$, and $10.1 \mu \mathrm{m}\left(29.1^{\circ}\right)$. This corresponds to zenith pwv values of $11.0 \mu \mathrm{m}, 6.9 \mu \mathrm{m}$, and $4.9 \mu \mathrm{m}$, respectively. The pwv overburden of SOFIA towards zenith can be calculated using the ATRAN (Atmospheric TRANsmission) model ${ }^{38}$. This yields $9.6 \mu \mathrm{m}\left(50.6^{\circ}\right), 7.3 \mu \mathrm{m}\left(38.3^{\circ}\right)$, and $5.5 \mu \mathrm{m}\left(29.1^{\circ}\right)$, which is in good agreement with the fitted values. Figure 7 displays the changes of the emission profile when the pwv is changed by $\pm 1 \mu \mathrm{m}$. This corresponds to a change of transmission of $\pm 7 \%$. It should be noted that the transmission affects the whole spectrum by the same factor. The uncertainty of the pwv is taken into account in the total uncertainty (see below).

For obtaining the radiances in Fig. 5 the pwv is deduced from fitting the saturated peak signals of all emission profiles during the flight and taking into account the elevation of the telescope. It yields an average value of $10.9 \pm 1.5 \mu \mathrm{m}$ pwv. This is larger than the deviation shown in Fig. 7 and corresponds to change of transmission by $\pm 12.5 \%$. The uncertainty is estimated from comparing the ATRAN calculation with the fitted pwv data. The single pwv value of $10.9 \mu \mathrm{m}$ has been used for all radiances in Fig. 5. The deviation of the measured radiances from the calculated ones is larger in the first $3000 \mathrm{~s}$ of the flight, because the deviation to the pwv of the fit in Fig. 2 is largest. In principle, it is possible to obtain a much better agreement between the measured and calculated radiances by fitting each spectrum with a specific pwv.

The total measurement uncertainty is determined by two parameters, namely the pwv and the reproducibility of the calibration procedure. The $1.5 \mu \mathrm{m}$ uncertainty of the pwv corresponds to an uncertainty of up to $12.5 \%$ of the radiance with the smaller uncertainty at smaller telescope elevation. Another source of uncertainty is the calibration procedure with the hot and cold loads. This includes the temperatures of the loads as well as gain drifts in the system and standing waves, which can change between calibration cycles. This uncertainty is $9 \%$. The total uncertainty of $15 \%$ is obtained from the root-sum-square of the pwv uncertainty and the calibration procedure uncertainty.

In Fig. 8a nine SABER atomic oxygen concentration profiles are shown. These spectra have been measured between 1:15 and 4:15 am on 14 January 2015 along the track shown in Fig. 1c. For comparison with the GREAT measurements we have computed the average profile of these nine profiles. This average profile is shown in Figs. 1a and $4 \mathrm{~b}$. The nighttime SABER measurements of the whole year 2015 (similar geolocation as GREAT: $20^{\circ}-40^{\circ}$ north latitude, $120^{\circ}-150^{\circ}$ west longitude) are visualized in Fig. $8 \mathrm{~b}$. The $1 \sigma$ variation is less than $30 \%$ of the mean at altitudes below $95 \mathrm{~km}$ and it increases above.

\section{Data availability}

The data for the atomic oxygen emission profiles measured with GRAT/SOFIA are available from the SOFIA data archive at https://irsa.ipac.caltech.edu/applications/sofia/? _action=layout.showDropDown\&visible $=$ true\&view $=$ Search (AOR IDs 83_0004_56, 98_0002_86, 98_0002_87,98_0002_88). The data for the atomic oxygen concentration profiles measured with SABER can be accessed at ftp://saber.gats-inc.com/Version2_0/ SABER_atox_Panka_etal_2018_GRL/SABER_o3p_oh_night_2015_v1.0.nc.

\section{Code availability}

The ATRAN code for calculating atmospheric transmission at the SOFIA flight altitude can be accessed at https://atran.arc.nasa.gov/cgi-bin/atran/atran.cgi. The code for
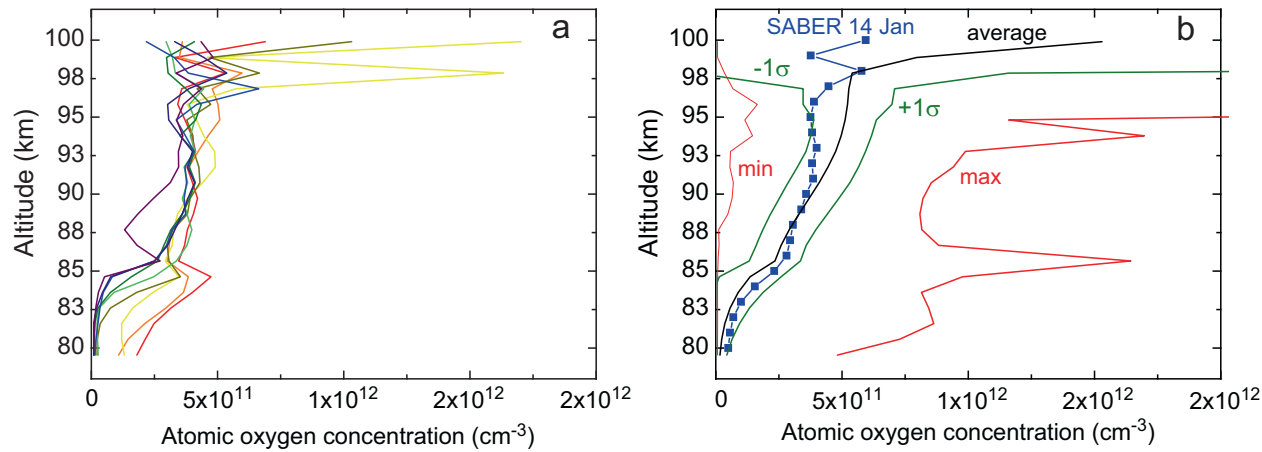

Fig. 8 Atomic oxygen concentration profiles measured with SABER. a Variation of the nine atomic oxygen concentration profiles which are used for the comparison with the GREAT measurements (each color refers to one measurement). b Average nighttime atomic oxygen in 2015 at a similar geolocation and time as the GREAT measurements (black line). The green lines are the $\pm 1 \sigma$ variations and the red lines are the maximum and the minimum atomic oxygen concentrations. The blue squares are the average of the profiles in Fig. 8a. This data is used for the comparison in the main text. 
calculating the NRLMSISE-00 profiles can be accessed at https://ccmc.gsfc.nasa.gov/ modelweb/models/nrlmsise00.php .

Received: 30 March 2020; Accepted: 14 December 2020; Published online: 26 January 2021

\section{References}

1. Mlynczak, M. G. \& Solomon, S. A detailed evaluation of the heating efficiency in the middle atmosphere. J. Geophys. Res. 98, 10,517-10,541 (1993).

2. Riese, M., Offermann, D. \& Brasseur, G. Energy released by recombination of atomic oxygen and related species at mesopause heights. J. Geophys. Res. 99, 14585-14593 (1994).

3. Feofilov, A. G. et al. $\mathrm{CO}_{2}\left(v_{2}\right)-\mathrm{O}$ quenching rate coefficient derived from coincidental SABER/TIMED and Fort Collins lidar observations of the mesosphere and lower thermosphere. Atmos. Chem. Phys. 12, 9013-9023 (2012).

4. Zink, L. R. et al. Atomic oxygen fine-structure splitting with tunable farinfrared spectroscopy. Astrophys. J. 371, L85-L86 (1991).

5. Ward, W. E. Tidal mechanisms of dynamical influence on oxygen recombination airglow in the mesosphere and lower thermosphere. Adv. Space Res. 21, 795-805 (1998).

6. Smith, A. K., Marsh, D. R., Mlynczak, M. G. \& Mast, J. C. Temporal variations of atomic oxygen in the upper mesosphere from SABER. J. Geophys. Res. 115, D18309 (2010)

7. Wu, D. L. et al. THz limb sounder (TLS) for lower thermospheric wind, oxygen density, and temperature. J. Geophys. Res. 121, 7301-7315 (2016).

8. Grossmann, K. U. \& Vollmann, K. Thermal infrared measurements in the middle and upper atmosphere. Adv. Space Res. 19, 631-638 (1997).

9. Offermann, D. et al. Cryogenic infrared spectrometers and telescopes for the atmosphere (CRISTA) experiment and middle atmosphere variability. J. Geophys. Res. 104, 16311-16325 (1999).

10. Grossmann, K. U., Kaufmann, M. \& Gerstner, E. A global measurement of lower thermosphere atomic oxygen densities. Geophys. Res. Lett. 27, 1387-1390 (2000).

11. Mlynczak, M. G. et al. Observations of the $\mathrm{O}\left({ }^{3} \mathrm{P}\right)$ fine structure line at $63 \mu \mathrm{m}$ in the upper mesosphere and lower thermosphere. J. Geophys. Res. 109, A12306 (2004).

12. Hedin, A. E. Extension of the MSIS thermosphere model into the middle and lower atmosphere. J. Geophys. Res. 96, 1159-1172 (1991).

13. Mlynczak, M. G. et al. Atomic oxygen in the mesosphere and lower thermosphere derived from SABER: algorithm theoretical basis and measurement uncertainty. J. Geophys. Res. 118, 5724-5735 (2013).

14. Zhu, Y. \& Kaufmann, M. Atomic oxygen abundance retrieved from SCIAMACHY hydroxyl night-glow measurements. Geophys. Res. Lett. 45, 1-9 (2018).

15. Russell, J. P. et al. Atomic oxygen profiles ( 80 to $115 \mathrm{~km}$ ) derived from Wind Imaging Interferometer/Upper Atmospheric Research Satellite measurements of the hydroxyl and greenline airglow: local time-latitude dependence. $J$. Geophys. Res. 110, D15305 (2005).

16. Kaufmann, M., Zhu, Y., Ern, M. \& Riese, M. Global distribution of atomic oxygen in the mesopause region as derived from SCIAMACHY O $\left({ }^{1} \mathrm{~S}\right)$ green line measurements. Geophys. Res. Lett. 41, 6274-6280 (2014).

17. Zhu, Y., Kaufmann, M., Ern, M. \& Riese, M. Nighttime atomic oxygen in the mesopause region retrieved from SCIAMACHY $\mathrm{O}\left({ }^{1} \mathrm{~S}\right)$ green line measurements and its response to solar cycle variations. J. Geophys. Res. 120, 9057-9073 (2015).

18. Sheese, P. E., McDade, I. C., Gattinger, R. L. \& Llewellyn, E. J. Atomic oxygen densities retrieved from Optical Spectrograph and Infrared Imaging System observations of $\mathrm{O}_{2} \mathrm{~A}$-band airglow emission in the mesosphere and lower thermosphere. J. Geophys. Res. 116, D01303 (2011).

19. Panka, P. A. et al. Atomic oxygen retrieved from SABER 2.0- and 1.6- $\mu \mathrm{m}$ radiances using new first principles nighttime $\mathrm{OH}(v)$ model. Geophys. Res. Lett. 45, 5798-5803 (2018).

20. Mlynczak, M. G., Hunt, L. A., Russell, J. M. III \& Marshall, B. T. Updated SABER night atomic oxygen and implications for SABER ozone and atomic hydrogen. Geophys. Res. Lett. 45, 5735-574 (2018).

21. Zhu, Y. \& Kaufmann, M. Consistent nighttime atomic oxygen concentrations from $\mathrm{O}_{2}$ A-band, $\mathrm{O}\left({ }^{1} \mathrm{~S}\right)$ green line, and $\mathrm{OH}$ airglow measurements as performed by SCIAMACHY. Geophys. Res. Lett. 46, 8536-8545 (2019).

22. Heyminck, S. et al. GREAT: the SOFIA high-frequency heterodyne instrument. Astron. Astrophys. 542, L1 (7 pages) (2012).

23. Ennico, K. et al. An overview of the Stratospheric Observatory for Infrared Astronomy since full operation capability. J. Astron. Instrum. 7, 1840012 (2018).

24. Rezac, L. et al. First detection of the $63 \mu \mathrm{m}$ atomic oxygen line in the thermosphere of Mars with GREAT/SOFIA. Astron. Astrophys. 580, L10 (2015).
25. Richter, H. et al. 4.7-THz local oscillator for the GREAT heterodyne spectrometer on SOFIA. IEEE Trans. Terahertz Sci. Technol. 5, 539-545 (2015).

26. Bulcha, B. T. et al. Design and characterization of 1.8-3.2 THz Schottky-based harmonic mixers. IEEE Trans. Terahertz Sci. Technol. 6, 737-746 (2016)

27. Hagelschuer, T. et al. A compact $4.75-\mathrm{THz}$ source based on a quantumcascade laser with a back-facet mirror. IEEE Trans. Terahertz Sci. Technol. 9, 606-612 (2019).

28. Zhang, Y. et al. Integrated wide-band CMOS spectrometer systems for spaceborne telescopic sensing. IEEE Trans. Circuits Syst. I 66, 1-11 (2019).

29. Wienold, M., Semenov, A., Richter, H. \& Hübers, H.-W. A balloon-borne $4.75 \mathrm{THz}$-heterodyne receiver to probe atomic oxygen in the atmosphere to appear in: Proceedings of the 45th International Conference on Infrared, Millimeter, and Terahertz Waves (IRMMW-THz) (Buffalo, NY, 2020, Publisher: IEEE).

30. Rea, S. P. et al. The low-cost upper-atmosphere sounder (LOCUS), Proceedings of the 26th International Symposium on Space Terahertz Technology. https://www.nrao.edu/meetings/isstt/papers/2015/2015000003. pdf (Smithsonian Astrophysical Observatory and Harvard College Observatory, Cambridge, MA, 2015).

31. Picone, J. M., Hedin, A. E., Drob, D. P. \& Aikin, A. C. NRLMSISE-00 empirical model of the atmosphere: statistical comparisons and scientific issues. J. Geophys. Res. 107, 1468 (2002).

32. Büchel, D. et al. 4.7-THz superconducting hot electron bolometer waveguide mixer. IEEE Trans. Terahertz Sci. Technol. 5, 207-214 (2015).

33. Klein, B. et al. High-resolution wide-band fast Fourier transform spectrometers. Astron. Astrophys. 542, L3 (2012).

34. Sharma, R., Zygelman, B., von Esse, F. \& Dalgarno, A. On the relationship between the population of the fine structure levels of the ground electronic state of atomic oxygen and the translational temperature. Geophys. Res. Lett. 21, 1731-1734 (1994)

35. Martin-Torres, F. J., Kutepov, A., Dudhia, A., Gusev, O. \& Feofilov, A. G. Accurate and fast computation of the radiative transfer absorption rates for the infrared bands in the atmosphere of Titan. Geophys. Res. Abstr. 5, 07735 (2003).

36. Kratz, D. P., Chou, M.-D., Yan, M.-H. \& Ho, C.-H. Minor trace gas radiative forcing calculations using the $\mathrm{k}$ distribution method with one parameter scaling. J. Geophys. Res. 103, 647-656 (1998).

37. Erickson, E. F. Effects of telluric water vapor on airborne infrared observations. Publ. Astron. Soc. Pac. 110, 1098-1105 (1998).

38. Lord, S. D. NASA Technical Memorandum 103957. https://atran.arc.nasa.gov/ cgi-bin/atran/atran.cgi (1992)

\section{Acknowledgements}

We acknowledge the work of the USRA and NASA staff of the Armstrong Flight Research Center in Palmdale and of the Ames Research Center in Mountain View, and the Deutsches SOFIA Institut. We thank the SABER team for producing the data and having it available to us. This work was funded in part by the German Federal Ministry of Research and Education (grant number 50 OK 1104). GREAT is a development by the MPI für Radioastronomie and the KOSMA/Universität zu Köln, in cooperation with the MPI für Sonnensystemforschung and the DLR Institut für Optische Sensorsysteme.

\section{Author contributions}

H.-W.H. conceived the idea. H.R. and H.-W.H. analyzed and interpreted the data. C.B., R.H., and H.W. contributed to the data analysis. C.B., R.G., H.-W.H., R.H., B.K., H.R., J.S., and H.W. carried out the observations. H.-W.H. provided overall guidance and wrote the paper, with contributions from all co-authors.

\section{Funding}

Open Access funding enabled and organized by Projekt DEAL.

\section{Competing interests}

The authors declare no competing interests.

\section{Additional information}

Correspondence and requests for materials should be addressed to H.-W.H.

Peer review information Primary handling editor: Joe Aslin.

Reprints and permission information is available at http://www.nature.com/reprints

Publisher's note Springer Nature remains neutral with regard to jurisdictional claims in published maps and institutional affiliations. 
(c) (i) Open Access This article is licensed under a Creative Commons Attribution 4.0 International License, which permits use, sharing, adaptation, distribution and reproduction in any medium or format, as long as you give appropriate credit to the original author(s) and the source, provide a link to the Creative Commons license, and indicate if changes were made. The images or other third party material in this article are included in the article's Creative Commons license, unless indicated otherwise in a credit line to the material. If material is not included in the article's Creative Commons license and your intended use is not permitted by statutory regulation or exceeds the permitted use, you will need to obtain permission directly from the copyright holder. To view a copy of this license, visit http://creativecommons.org/ licenses/by/4.0/.

(C) The Author(s) 2021 